\title{
Afferent baroreflex failure in familial dysautonomia
}

Lucy Norcliffe-Kaufmann, $\mathrm{PhD}$

Felicia Axelrod, MD Horacio Kaufmann, MD, FAAN

Address correspondence and reprint requests to Dr. Horacio Kaufmann, New York University School of Medicine, 530 First Avenue Suite 9Q, New York, NY 10016

Horacio.kaufmann@nyumc.org

\section{ABSTRACT}

Background: Familial dysautonomia (FD) is due to a genetic deficiency of the protein IKAP, which affects development of peripheral neurons. Patients with FD display complex abnormalities of the baroreflex of unknown cause.

Methods: To test the hypothesis that the autonomic phenotype of FD is due to selective impairment of afferent baroreceptor input, we examined the autonomic and neuroendocrine responses triggered by stimuli that either engage (postural changes) or bypass (cognitive/emotional) afferent baroreflex pathways in 50 patients with FD and compared them to those of normal subjects and to those of patients with pure autonomic failure (PAF), a disorder with selective impairment of efferent autonomic neurons.

Results: During upright tilt, in patients with FD and in patients with PAF blood pressure fell markedly but the heart rate increased in PAF and decreased in FD. Plasma norepinephrine levels failed to increase in both groups. Vasopressin levels increased appropriately in patients with PAF but failed to increase in patients with FD. Head-down tilt increased blood pressure in both groups but increased heart rate only in patients with FD. Mental stress evoked a marked increase in blood pressure and heart rate in patients with FD but little change in those with PAF.

Conclusion: The failure to modulate sympathetic activity and to release vasopressin by baroreflex-mediated stimuli together with marked sympathetic activation during cognitive tasks indicate selective failure of baroreceptor afference. These findings indicate that IKAP is critical for the development of afferent baroreflex pathways and has therapeutic implications in the management of these patients. Neurology ${ }^{\circledR}$ 2010;75:1904-1911

GLOSSARY

FD = familial dysautonomia; FVR = forearm vascular resistance; PAF = pure autonomic failure.

Familial dysautonomia (FD) is a hereditary disease caused by a mutation affecting the protein IKAP. ${ }^{1,2}$ Patients with FD have a complex neurologic phenotype, first described by Riley et al. ${ }^{3}$ Their original report featured 5 children who responded to mild anxiety with hypertension, tachycardia, red blotching of the skin, and diaphoresis. Interestingly, the patients also had postural hypotension and did not complain of pain when their feet were immersed in ice-cold water. Neuropathology studies in samples from patients with FD showed reduced number of primary sensory neurons in dorsal root ganglia ${ }^{4}$ and fiber loss in spinothalamic and spinocerebellar tracts as well as in posterior columns of the spinal cord..$^{5}$ These findings provided an explanation for the impaired pain and temperature perception as well as the characteristic decreased/absent myotatic reflexes and gait ataxia.

The autonomic phenotype of these patients, particularly their blood pressure abnormalities, have, however, remained unexplained. Subjects have supine hypertension and severe orthostatic hypotension, which could be perhaps due to a presumed decrease in the number of sympathetic neurons. ${ }^{6-8}$ Yet patients with FD also have recurrent episodes of hypertension with tachycardia, which are frequently associated with skin flushing and diaphoresis and suggest that

From the Dysautonomia Center, New York University School of Medicine, New York, NY.

Study funding: Supported by the Dysautonomia Foundation, Inc., and the National Institutes of Health (R01-FD003731-01 and U54-NS065736-01).

Disclosure: Author disclosures are provided at the end of the article. 
sympathetic neurons can be activated and are functional. We noted that these episodes resemble those described in the very rare patients in whom afferents fibers from baroreceptor in the carotid sinus and aortic arch traveling in the vagus and glossopharyngeal nerves have been damaged by tumors, surgery, or radiotherapy. ${ }^{9,10}$ Thus, we postulated that the blood pressure abnormalities of patients with FD might be due to a selective impairment of the afferent neurons of the baroreflex.

To test this hypothesis, we analyzed the autonomic and neuroendocrine responses triggered by stimuli that either engage or bypass afferent baroreflex pathways and compared them to those of normal subjects and to those of patients with selective efferent autonomic dysfunction due to pure autonomic failure (PAF). ${ }^{11,12}$

METHODS Patients. Fifty patients with FD (age $22 \pm 2$ years, 23 men), confirmed by genetic testing, ${ }^{13} 10$ patients with PAF (age $64 \pm 4$ years, 5 men), ${ }^{11}$ and 12 normal controls (age $26 \pm 2$ years, 5 men) participated in the study. Although matched for age, patients with FD were shorter (height: FD $146 \pm 2$ vs controls $166 \pm 1 \mathrm{~cm}, p<0.001)$ and lighter (weight: FD $40 \pm 2$ vs controls $58 \pm 10 \mathrm{~kg}, p<0.001$ ) than normal controls, a well-described abnormality in these patients. The diagnosis of PAF was based on the presence of severe orthostatic hypotension, low plasma norepinephrine concentration, no antibody titers to nicotinic ganglionic cholinergic receptors, absent blood pressure overshoot during phase IV of a Valsalva maneuver, no somatic neurologic deficits, and a disease duration of 5 years or more. ${ }^{14}$ Because FD is a congenital disorder and $\mathrm{PAF}$ is a neurodegenerative disorder, patients with FD were younger than patients with PAF. All procedures were approved by the Institutional Review Board of New York University and informed consent was obtained from all subjects.

Laboratory protocol. An IV catheter was inserted into the left cubital vein and the subject was transferred to a tilt table. After a period of at least 20 minutes of supine rest when the patient appeared relaxed, blood pressure, heart rate, forearm blood flow velocity, and end-tidal $\mathrm{CO}_{2}$ levels were recorded during spontaneous breathing for 5 minutes. Blood samples were drawn for the measurement of plasma norepinephrine and vasopressin levels while supine. The subject was then tilted passively to a 30-degree and then to a 60-degree angle, where they remained immobile with footplate support for at least 10 minutes. After 10 minutes and while still tilted, a second set of blood samples was drawn for the measurement of plasma norepinephrine and vasopressin levels. The subject was then returned to the supine position and after 5 minutes the table was tilted 15 degrees head-down for 5 minutes and then returned to the horizontal position. After at least 10 minutes of relaxation subjects performed a difficult mental arithmetic stressor task (serial subtractions of 7). Subjects were repeatedly urged to respond with sequential answers as rapidly as possible. Continued stress was applied in this manner for 3 minutes. Patients with PAF and controls also performed a standardized Valsalva maneuver by blowing into a mouthpiece for 15 seconds maintaining a pressure of $40 \mathrm{~mm} \mathrm{Hg}$. Patients with FD could not perform Valsalva maneuver because they were unable to maintain adequate expiratory pressure.

In 4 patients with FD, plasma norepinephrine was obtained during a typical dysautonomic crisis (hypertension, tachycardia, flushing of the skin, nausea and retching), 20 minutes after symptoms began and repeated on a separate occasion after the symptoms had resolved.

The relationship between changes in heart rate and changes in systolic blood pressure, induced by head-up and head-down body tilt (in patients) and by a Valsalva maneuver (in normal subjects), expressed as beats per $\mathrm{min} / \mathrm{mm} \mathrm{Hg}$ was used as a measure of cardiac baroreflex gain. The gain of the reflex was obtained by computing the slope of the regression line relating changes in systolic pressure to changes in RR intervals, as previously described. ${ }^{15}$ Computed slopes were then averaged to obtain the baroreflex slope.

Baroreflex control of forearm vascular resistance (FVR) was determined during graded gravitational stress, including 30 and 60 degrees head-up and 15 degrees head-down tilt. Changes in FVR were derived from the changes in brachial artery blood flow velocity and mean arterial pressure, ${ }^{16}$ with the assumption that brachial artery diameter remains relatively constant. ${ }^{17}$ The relationship between percentage changes in FVR and the gravity vector was used as a measure of vascular baroreflex sensitivity and was expressed as $\Delta \% / \mathrm{m} \cdot \mathrm{sec}^{2}$.

Atropine test. In one patient with FD, we used parasympathetic blockade with atropine to further define the heart rate changes induced by posture. Blood pressure and heart rate were measured supine and standing before and after the administration of $40.01 \mathrm{mg} / \mathrm{kg}$ IV boluses of atropine to achieve complete parasympathetic blockade.

Measurements. Beat-to-beat blood pressure was measured in the right finger using plethysmography (Finometer/Finapres Medical Systems BV, Amsterdam, the Netherlands). Intermittent blood pressure recordings were taken in the left arm with an automatic sphygmomanometer (Colin Press-Mate 7800, San Antonio, TX). Right brachial artery flow velocity was measured with a $4 \mathrm{mHz}$ Doppler probe (Multi-Dop T, Singen, Germany), which was locked in place with a series of clamps fixed to the armrest to maintain a constant angle of insonation. End-tidal $\mathrm{CO}_{2}$ levels were sampled through a nasal cannula and measured using infrared analysis (Ohmeda 5200, Englewood, CO). RR intervals were recorded from 3 precordial electrodes. All signals were acquired and digitized (DA-720, Akron, OH). Plasma norepinephrine was measured using high-performance liquid chromatography with electrochemical detection. Plasma concentration of vasopressin was measured using radioimmunoassay (ARUP Laboratories, Salt Lake City, UT).

RESULTS Cardiovascular and neuroendocrine responses to baroreflex changes. To test the integrity of afferent and efferent baroreceptor pathways in patients with FD, we examined their autonomic and neuroendocrine responses while supine, and during stimulation with head-up and head-down passive body tilt. Head-up and head-down tilt decreases and increases, respectively, cardiac filling and in normal 
Figure 1 Heart rate (HR), blood pressure (BP), plasma norepinephrine (NE), and plasma vasopressin concentration while in the supine position and during head-up and head-down tilt in patients with familial dysautonomia (FD), patients with pure autonomic failure (PAF), and normal controls
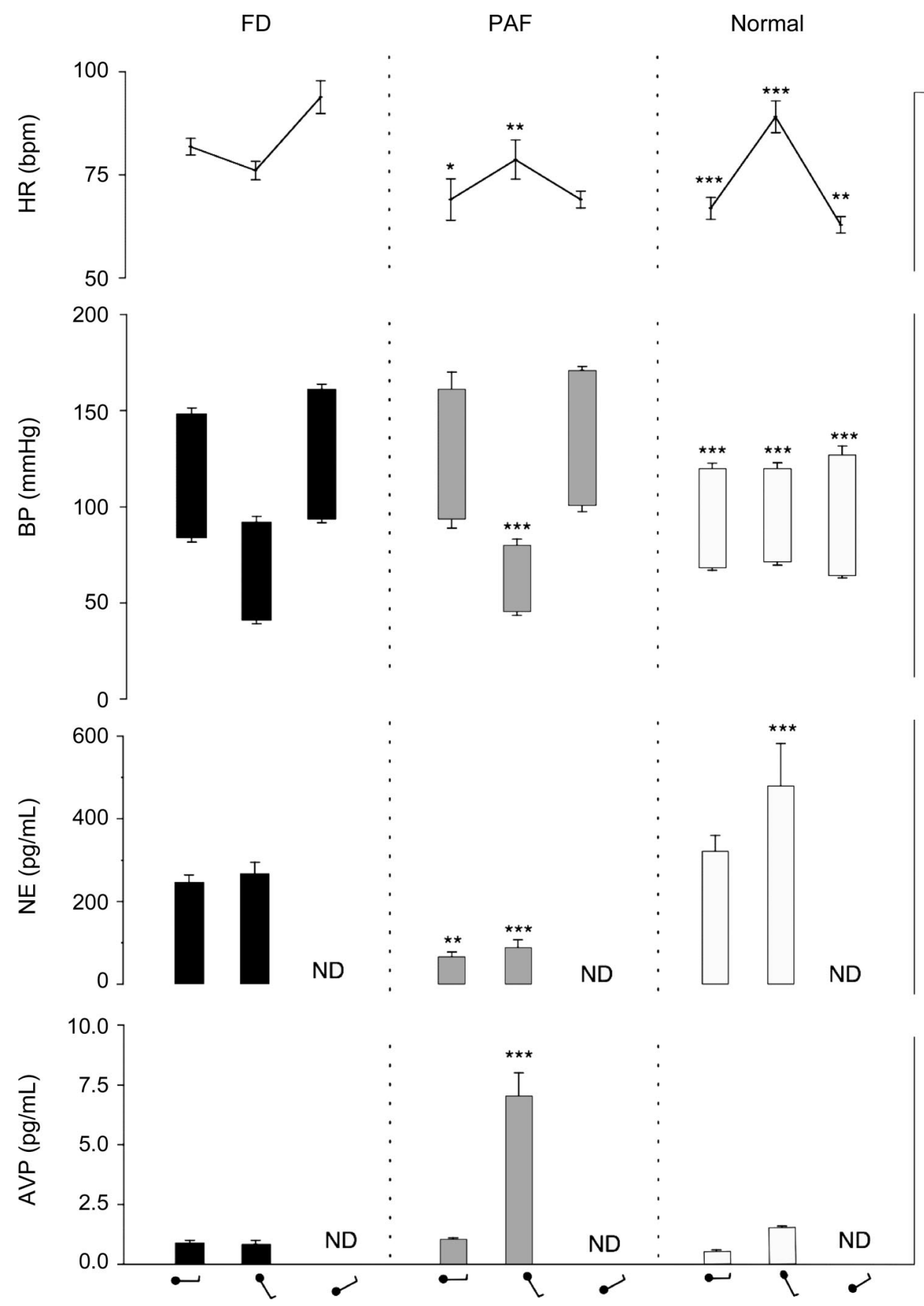

$\mathrm{AVP}=$ arginine vasopressin; ND = not done. Figurine denotes position on the tilt table (supine, head-up and head-down tilt). ${ }^{* * *} p<0.001,{ }^{* *} p<0.02,{ }^{*} p<0.05$.

subjects results in opposite changes in afferent baroreceptor activity. The patients' responses were compared to those of normal subjects and of patients with PAF, who have a selective deficiency in efferent sympathetic pathways. ${ }^{11,12}$

Blood pressure and plasma norepinephrine while supine. Compared to control subjects, patients with FD, like patients with PAF, had elevated blood pres- sure when supine (figure 1). However, plasma norepinephrine concentration in patients with FD $(262 \pm 33 \mathrm{pg} / \mathrm{mL})$ was similar to that in normal subjects $(315 \pm 45 \mathrm{pg} / \mathrm{mL})$ and significantly higher than the low levels characteristic of patients with PAF $(63 \pm 14 \mathrm{pg} / \mathrm{mL}, p<0.02)$, suggesting intact sympathetic neurons in patients with FD. While supine, heart rate was significantly higher in patients 

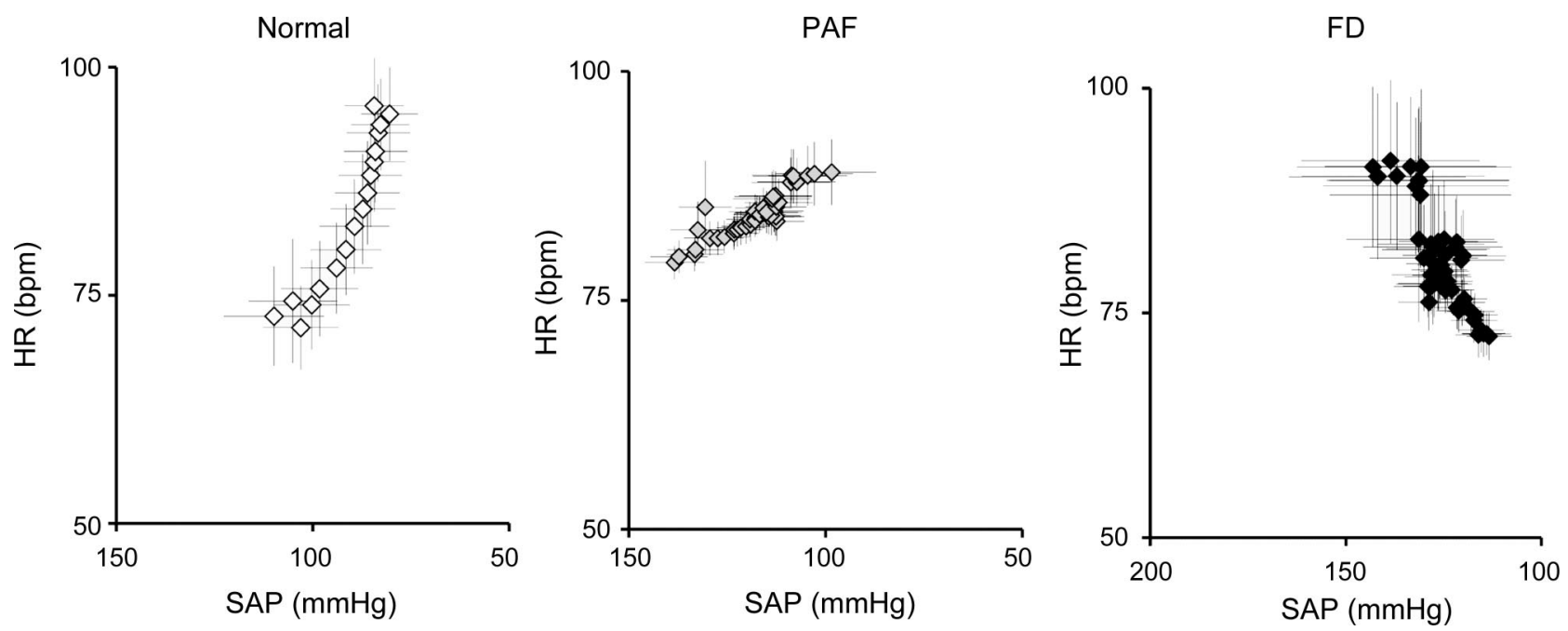

Baroreflex function was determined by the relationship between blood pressure and heart rate during the first 40 beats of upright tilt. Each value represents the average of 50 subjects. Quantification of these data showed that cardiac baroreflex sensitivity was $-0.87 \mathrm{bpm} / \mathrm{mm} \mathrm{Hg} \mathrm{in} \mathrm{controls}(y=-0.87 \mathrm{x}+$ $\left.163, R^{2}=0.88, \mathrm{p}<0.001\right),-0.24 \mathrm{bpm} / \mathrm{mm} \mathrm{Hg}$ in PAF $\left(\mathrm{y}=-0.24 \mathrm{x}+113, R^{2} 0.81, \mathrm{p}<0.001\right)$, and $+0.71 \mathrm{bpm} / \mathrm{mm} \mathrm{Hg}$ in FD $\left(\mathrm{y}=+0.71 \mathrm{x}-8, R^{2}=0.74\right.$, $p<0.01) . F D=$ familial dysautonomia; $H R=$ heart rate; $P A F=$ pure autonomic failure; $S A P=$ systolic arterial pressure.

with FD $(82 \pm 2$ beats/min) than in patients with $\operatorname{PAF}(69 \pm 5 \mathrm{beat} / \mathrm{min}, p<0.02)$ and in the normal controls $(67 \pm 3, p<0.01)$.

Changes in plasma norepinephrine and blood pressure. As shown in figure 1, during upright tilt, blood pressure fell markedly in patients with FD and patients with PAF. Yet in both groups of patients and unlike controls, plasma norepinephrine levels failed to increase, indicating lack of sympathetic activation during the marked hypotension. As expected, normal subjects had little change in blood pressure both during upright tilt as well as during head-down tilt. In marked contrast, both in patients with FD and patients with PAF, head-down tilt induced an increase in systolic blood pressure of more than $10 \mathrm{~mm} \mathrm{Hg}$, compared to the blood pressure level when supine, demonstrating, like during upright tilt, inappropriate baroreflex function.

Changes in FVR. To test directly whether changes in baroreceptor loading modulate sympathetic efferent activity to the vasculature in patients with $\mathrm{FD}$, we monitored their FVR during gravitational changes. During head-up tilt, contrary to normal subjects in whom FVR appropriately increased, FVR decreased in patients with FD, confirming the lack of baroreflex-mediated sympathetic activation suggested by the fall in blood pressure. Similarly, when venous return to the heart increased during headdown tilt, while in normal subjects FVR appropriately decreased indicating a reduction in baroreflexmediated sympathetic activity, in patients with FD, forearm vascular resistance, like the blood pressure, increased, indicating absent baroreflex inhibition of sympathetic outflow to the vasculature. Consequently, the relationship between gravitational forces and FVR in patients with FD (slope, $-68 \Delta \%$ / $\mathrm{m} . \mathrm{sec}^{2}$ ) was the reverse to that of control subjects (slope, $53 \Delta \% / \mathrm{m} \cdot \sec ^{2}$ ).

Changes in heart rate. While orthostatic stress of upright tilt similarly decreased blood pressure in patients with FD and patients with PAF, it had opposite effects on their heart rate. Heart rate increased in patients with PAF, albeit less than in normal subjects, but it unexpectedly decreased in patients with FD (figure 1). Similarly, head-down tilt also caused different changes in the heart rate of the 2 groups of patients. In response to the increase in cardiac filling, heart rate did not change in patients with PAF but in patients with FD it increased by an average of 12 beats (figure 1).

Quantification of the relationship between blood pressure and heart rate is shown in figure 2. In control subjects, there was the expected inverse relationship between blood pressure and heart rate characteristic of the normal baroreflex. In patients with PAF, there was a similar relationship, but as previously noted, ${ }^{19}$ the slope was markedly less steep due to impaired efferent innervation of the heart. However, in patients with FD the relationship between blood pressure and heart rate was reversed, resulting in a positive correlation, indicating completely absent baroreflex modulation of heart rate.

The dramatic decrease in heart rate during upright stimulation in patients with FD led us to consider whether it could be mediated by an increase in 
parasympathetic activity. To exclude this possibility, we blocked parasympathetic signaling by atropine administration during orthostatic challenge in a patient with FD. Following atropine, heart rate increased from 106 to $116 \mathrm{bpm}$ while supine (16\%), indicating low resting parasympathetic tone. However, subsequent orthostatic stress after atropine lowered blood pressure but the patient's heart rate again decreased, 7 beats, from 116 to $109 \mathrm{bpm}$, indicating that the bradycardia was not due to increased parasympathetic signaling. The same dose of atropine given to a control normal subject while supine increased heart rate from 60 to $105 \mathrm{bpm}(92 \%)$ and after standing, heart rate increased further from 105 to $111 \mathrm{bpm}$.

Vasopressin response to hypotension. Baroreflex release of vasopressin, unlike norepinephrine release from sympathetic efferents, only requires intact afferent baroreceptor pathways. ${ }^{12}$ Upon standing, normal subjects had an appropriately modest but detectable increase in plasma vasopressin (figure 1), but in patients with PAF, upright tilt resulted in a marked increase in plasma vasopressin, as would be expected from the profound stimulation of the baroreflex by the marked decrease in blood pressure. In contrast, despite having a comparable fall in blood pressure to that of patients with PAF, patients with FD had no increase in plasma vasopressin during orthostatic stress (figure 1). The inability of the patients with FD to secrete vasopressin during marked hypotension indicates that afferent baroreceptor pathways are affected.

Stimulation of sympathetic activity independently of baroreceptor afferents. To further explore the integrity of the sympathetic efferent neurons in patients with FD, we studied the effect of cognitive tasks and emotional arousal on their blood pressure and heart rate. Both situations activate forebrain cortical neurons and increase sympathetic activity through direct connections to the hypothalamus and ventrolateral medulla independently of baroreceptor afference. ${ }^{18}$

Cognitive task. Cognitive tasks induced marked increases in heart rate and blood pressure in patients with FD. The systolic blood pressure increased by $32 \pm 3 \mathrm{~mm} \mathrm{Hg}$ and the heart rate by $11 \pm 2$ beats/ $\min (\mathrm{n}=15 ; p<0.01$ for both). In marked contrast, patients with PAF had very small responses to identical stimuli; systolic blood pressure increased by $6 \pm 2 \mathrm{~mm} \mathrm{Hg}$, and heart rate increased $4 \pm 5$ beats/ $\min (\mathrm{n}=6, p<0.05$ for both).

Emotional arousal. Previous clinical observations indicated that emotionally charged situations caused increases in blood pressure and heart rate in patients with FD. We thus aimed to record these changes in several patients and figure 3 shows 3 different examples. In one patient, following a phone call from a friend, blood pressure quickly increased from 150/85 to $260 / 180 \mathrm{~mm} \mathrm{Hg}$ and the heart rate from 70 to 150 beats/min. In another patient, an argument with his mother increased the blood pressure from 130/75 to $220 / 145 \mathrm{~mm} \mathrm{Hg}$ and the heart rate from 100 to 140 beats/min. Finally, when the study nurse entered the room to draw blood from the third patient, standing blood pressure increased from $85 / 60 \mathrm{~mm}$ $\mathrm{Hg}$ to $140 / 100 \mathrm{~mm} \mathrm{Hg}$.

Changes in plasma levels of norepinephrine during crises. Finally, to obtain neurotransmitter evidence that sympathetic neurons are properly activated in patients with FD, we measured norepinephrine plasma levels during crises triggered by emotionally charged situations. Figure 4 shows that in addition to a marked increase in blood pressure and heart rate during emotionally triggered crises, plasma norepinephrine levels increased from $152 \pm 74$ to $780 \pm 260$ $\mathrm{pg} / \mathrm{mL}(\mathrm{n}=4 ; p<0.04)$.

DISCUSSION The results of this study show that patients with FD have impaired baroreceptor afference but preserved sympathetic efferent neurons (figure 5). Gravitational stimuli, sensed by baroreceptors in the vasculature, failed to elicit appropriate adjustments of heart rate, forearm vascular resistance, and plasma norepinephrine concentration (figure 1). This defect was not due to impaired efferent neurons because cognitive tasks and emotional arousal, stimuli that activate sympathetic neurons in the hypothalamus and medulla independently of baroreceptor afference, dramatically increased blood pressure, heart rate, and circulating norepinephrine levels (figures 1 and 2). Hence, patients with FD have a selective defect in afferent baroreceptor neurons. Indeed, the afferent baroreflex defect in these patients was confirmed by the failure to release vasopressin during pronounced hypotension. Of the many responses to baroreflex stimulation, vasopressin secretion is likely the only one that is solely dependent on afferent pathways. ${ }^{12}$ This is why patients with PAF who are unable to release norepinephrine and to maintain blood pressure upon standing had a markedly elevated plasma vasopressin during upright tilt (see figure 1). In contrast, patients with FD had an equally severe drop in blood pressure but were unable to increase their plasma concentration of vasopressin.

The cell bodies of afferent baroreceptor neurons are in the nodose and petrosal ganglia of the glossopharyngeal and vagus nerves. These neurons are the functional equivalent of primary sensory neurons in the dorsal root ganglia, a neuronal population markedly depleted in patients with FD. 
FD patient $A$

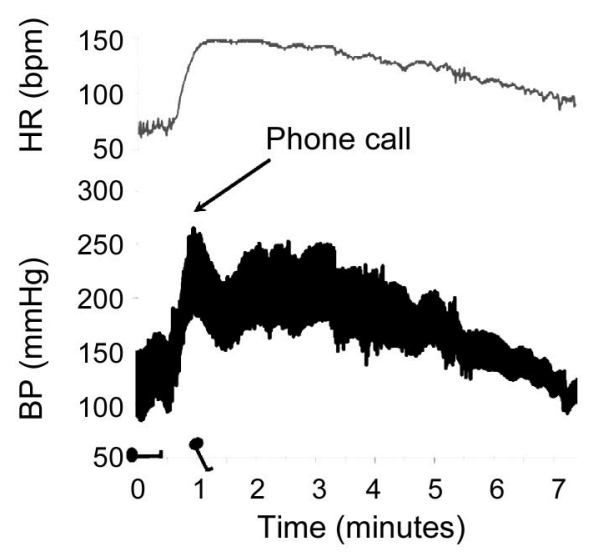

FD patient C

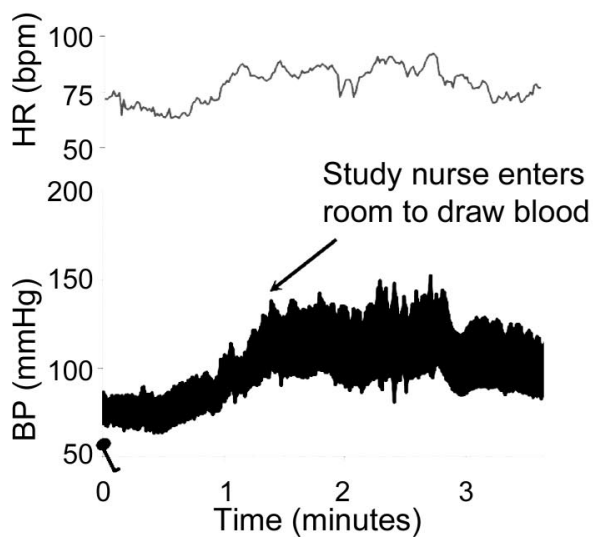

FD patient $B$

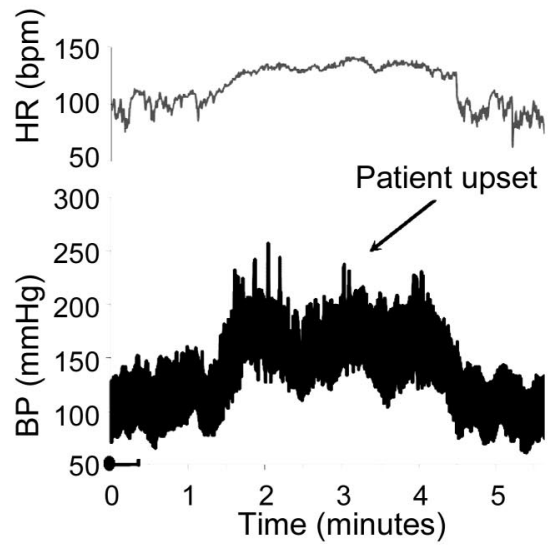

Response to cognitive stimuli

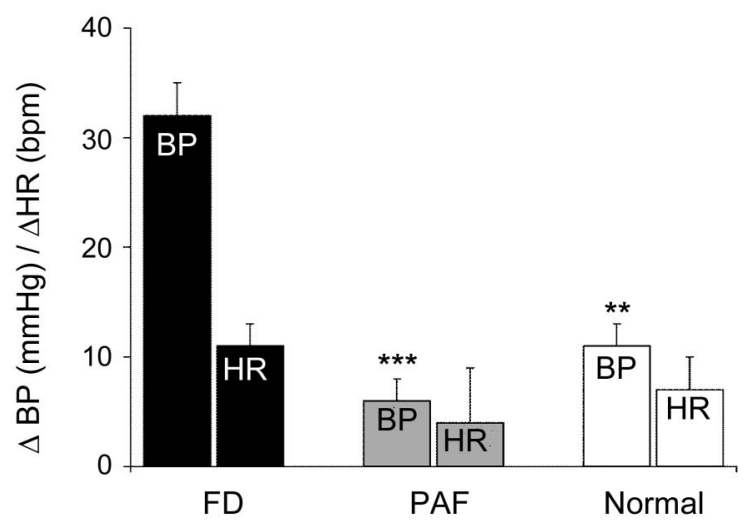

(A) Beat-to-beat BP and HR in a patient with familial dysautonomia (FD) who received an unexpected phone call from a friend. Her BP and HR rose in parallel. She was then tilted to the upright position $(\min 1)$. BP initially fell, but quickly rose again. (B) BP and HR in a boy with FD during an argument with his mother. (C) BP and HR in a patient with FD when the study nurse enters the room to draw blood. (D) Changes in BP and HR in patients with FD, pure autonomic failure (PAF), and normal controls induced by a cognitive stimulus (mental arithmetic).

The mechanism that controls heart rate in patients with FD is intriguing. The normal changes in heart rate induced by posture were reversed (figure 1). These heart rate changes are unlikely to be neurally mediated, as they were the opposite of the expected baroreflex-mediated changes. Moreover, muscarinic blockade with atropine failed to prevent the slowing of the heart during orthostasis, indicating that this relative bradycardia was not caused by an increase in parasympathetic signaling to the sinoatrial node. For similar reasons, the tachycardia during head-down tilt is unlikely to be mediated by changes in autonomic activity. Instead, the control of heart rate in FD appears dependent on distension of the atria; it increased during head-down tilt and decreased during head-up tilt. These heart rate responses closely resemble those observed in isolated right atrium in both acutely and chronically decentralized heart preparations in which changes in chamber pressure, or myocardial stretch, regulate the cardiac pacemaker. ${ }^{19-24}$ No neurons participate in this intrinsic control of heart rate that starts in stretch-activated ion channels of atrial cells. ${ }^{25-27}$

This "intrinsic" heart rate regulating mechanism has not been described in patients with acquired baroreflex failure. Perhaps this is because other mechanisms that modulate autonomic responses through vestibular and proprioceptive afferents are preserved in acquired baroreflex failure but are likely affected in FD. ${ }^{6,7}$ Indeed, compensation by these alternative afferent pathways may explain why many patients with acquired baroreflex failure do not have postural hypotension. ${ }^{9,10}$

The disabling crises of patients with FD (figure 4) are readily explained by afferent baroreceptor denervation. Indeed, very similar episodes with headache, palpitations, flushing of the skin, and psychomotor agitation occur in patients with acquired afferent baroreflex fail- 


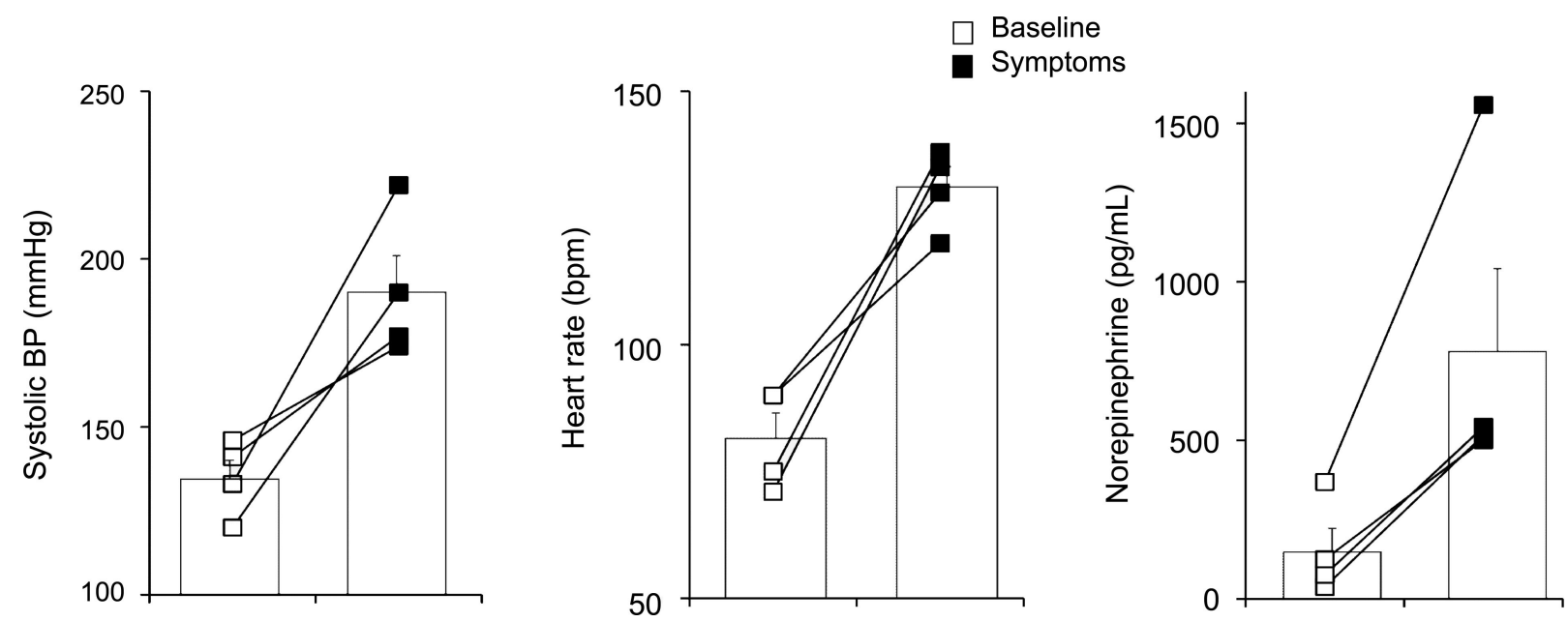

Left bar charts show systolic BP, middle bar chart shows heart rate, and right bar chart shows plasma norepinephrine concentration at baseline (open squares) and during a typical crisis provoked by an emotionally charged situation (black squares).

ure. ${ }^{9}$ In these subjects, similar to what occurs in FD, sympathetic outflow to the vasculature becomes very responsive to emotional influences. ${ }^{18}$ Because surges in sympathetic activity are not restrained by the normal baroreceptor feedback, the increase in blood pressure and heart rate during emotional arousal is amplified and prolonged (figure 3)..$^{3,28}$ Moreover, as neuropathology $y^{6,7}$ and imaging ${ }^{29}$ studies suggest reduced number of sympathetic neurons, it is likely that patients with FD also have vascular "denervation supersensitivity," 30 further increasing the hypertensive responses to sympathetic surges.

Afferent baroreflex failure explains previously reported findings in patients with FD, including the parallel increase in blood pressure and heart rate observed by Smith and Dancis ${ }^{31}$ during norepinephrine infusion, the sharp rise in blood pressure and heart rate when venous return was increased during a

\section{Figure 5 Afferent and efferent pathways involved in cardiovascular control in patients with familial} dysautonomia and patients with pure autonomic failure

Familial dysautonomia

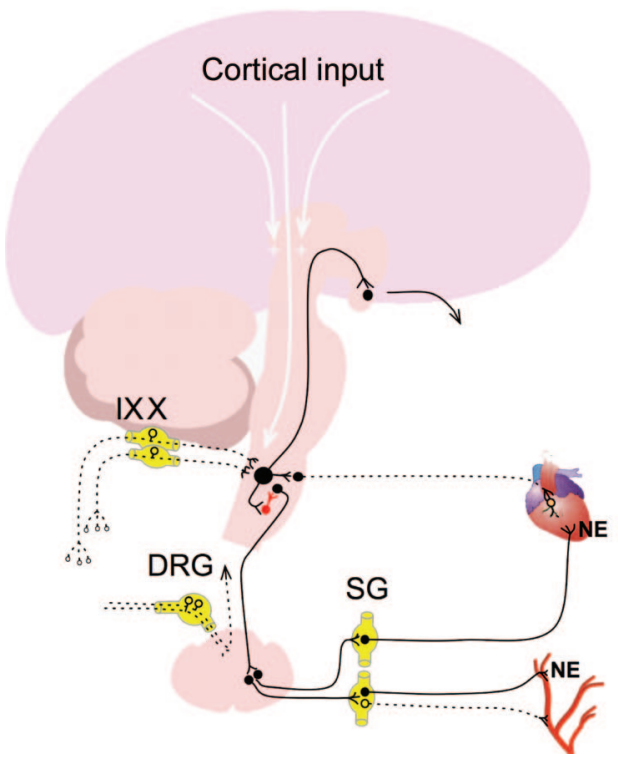

Pure autonomic failure

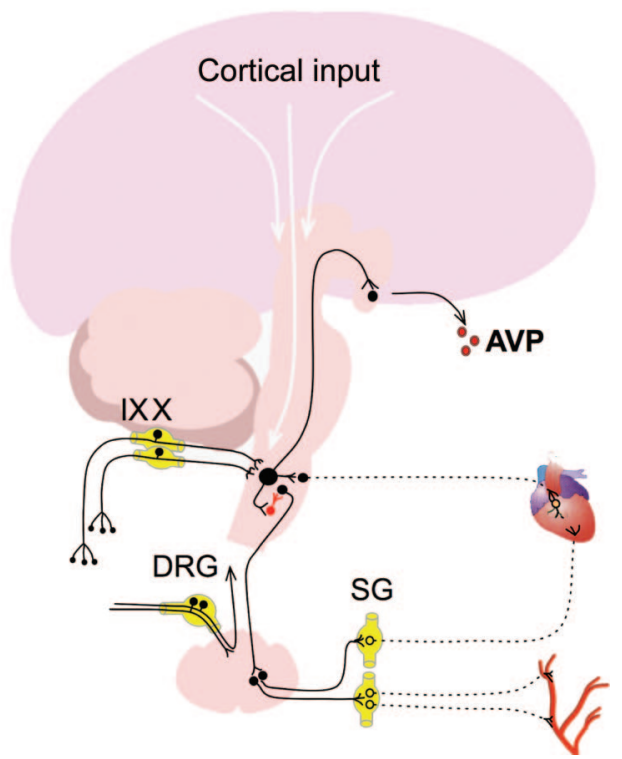

Dotted line indicates site of abnormality. IX = glossopharyngeal neurons with cell bodies in the petrosal ganglia relaying information from the carotid baroreceptors; $X$ = vagal neurons with cell bodies in the nodose ganglia relaying information from baroreceptors in the aortic arch; AVP = arginine vasopressin; DRG = dorsal root ganglia with cell bodies of primary sensory neurons; $\mathrm{NE}=$ norepinephrine; $\mathrm{SG}$ = sympathetic ganglia. 
blood transfusion, ${ }^{32}$ and the lack of heart rate modulation following direct carotid baroreceptor stimulation with neck collars. ${ }^{33}$

\section{ACKNOWLEDGMENT}

The authors thank Dr. Juan Oliver for comments on the manuscript.

\section{DISCLOSURE}

Dr. Norcliffe-Kaufmann receives research support from the NIH (U54NS065736 [Assistant Research Scientist]) and the FDA (FD-R3731-01 [Assistant Research Scientist]). Dr. Axelrod receives research support from the Dysautonomia Foundation, Inc. Dr. Kaufmann serves on a scientific advisory board for Chelsea Therapeutics; serves as Editorin-Chief of Clinical Autonomic Research; and receives research support from the NIH (U54NS065736 [PI]), the FDA (FD-R-3731-01 [PI]), and the Dysautonomia Foundation, Inc.

Received May 20, 2010. Accepted in final form August 10, 2010.

\section{REFERENCES}

1. Slaugenhaupt SA, Blumenfeld A, Gill SP, et al. Tissuespecific expression of a splicing mutation in the IKBKAP gene causes familial dysautonomia. Am J Hum Genet 2001;68:598-605.

2. Anderson SL, Coli R, Daly IW, et al. Familial dysautonomia is caused by mutations of the IKAP gene. Am J Hum Genet 2001;68:753-758.

3. Riley CM, Day RA, Greeley DM, Landford WS. Central autonomic dysfunction with defective lacrimation: I: report of five cases. Pediatrics 1949;3:468-478.

4. Pearson J, Pytel BA, Grover-Johnson N, Axelrod F, Dancis J. Quantitative studies of dorsal root ganglia and neuropathologic observations on spinal cords in familial dysautonomia. J Neurol Sci 1978;35:77-92.

5. Brown WJ, Beauchemin JA, Linde LM. A neuropathological study of familial dysautonomia (Riley-Day syndrome) in siblings. J Neurol Neurosurg Psychiatry 1964;27:131-139.

6. Dyck PJ, Kawamura Y, Low PA, Shimono M, Solovy JS The number and sizes of reconstructed peripheral autonomic, sensory and motor neurons in a case or dysautonomia. J Neuropathol Exp Neurol 1978;37:741-755.

7. Pearson J, Pytel BA. Quantitative studies of sympathetic ganglia and spinal cord intermedio-lateral gray columns in familial dysautonomia. J Neurol Sci 1978;39:47-59.

8. Pearson J, Brandeis L, Goldstein M. Tyrosine hydroxylase immunoreactivity in familial dysautonomia. Science 1979; 206:71-72.

9. Robertson D, Hollister AS, Biaggioni I, Netterville JL, Mosqueda-Garcia R, Robertson RM. The diagnosis and treatment of baroreflex failure. N Engl J Med 1993;329: $1449-1455$.

10. Biaggioni I, Whetsell WO, Jobe J, Nadeau JH. Baroreflex failure in a patient with central nervous system lesions involving the nucleus tractus solitarii. Hypertension 1994; 23:491-495.

11. Hague K, Lento P, Morgello S, Caro S, Kaufmann H. The distribution of Lewy bodies in pure autonomic failure: autopsy findings and review of the literature. Acta Neuropathol 1997;94:192-196.

12. Kaufmann H, Oribe E, Miller M, Knott P, WiltshireClement M, Yahr MD. Hypotension-induced vasopressin release distinguishes between pure autonomic failure and multiple system atrophy with autonomic failure. Neurology 1992;42:590-593.
13. Blumenfeld A, Slaugenhaupt SA, Liebert CB, et al. Precise genetic mapping and haplotype analysis of the familial dysautonomia gene on human chromosome 9q31. Am J Hum Genet 1999;64:1110-1118.

14. Kaufmann H, Biaggioni I. Autonomic failure in neurodegenerative disorders. Semin Neurol 2003;23:351-363.

15. Tellez MJ, Norcliffe-Kaufmann LJ, Lenina S, Voustianiouk A, Kaufmann H. Usefulness of tilt-induced heart rate changes in the differential diagnosis of vasovagal syncope and chronic autonomic failure. Clin Auton Res 2009;19:375-380.

16. Norcliffe-Kaufmann LJ, Kaufmann H, Hainsworth R. Enhanced vascular responses to hypocapnia in neurally mediated syncope. Ann Neurol 2008;63:288-294.

17. Herrington DM, Fan L, Drum M, et al. Brachial flowmediated vasodilator responses in population-based research: methods, reproducibility and effects of age, gender and baseline diameter. J Cardiovasc Risk 2001;8:319-328.

18. Fagius J, Wallin BG, Sundlof G, Nerhed C, Englesson S. Sympathetic outflow in man after anaesthesia of the glossopharyngeal and vagus nerves. Brain 1985;108:423-438.

19. Blinks JR. Positive chronotropic effect of increasing right atrial pressure in the isolated mammalian heart. Am J Physiol 1956;186:299-303.

20. Pathak CL. Effects of changes in intraluminal pressure on inotropic and chronotropic responses of isolated mammalian hearts. Am J Physiol 1958;194:197-199.

21. Brooks CM, Lu HH, Lange G, Mangi R, Shaw RB, Geoly $\mathrm{K}$. Effects of localized stretch of the sinoatrial node region of the dog heart. Am J Physiol 1966;211:1197-1202.

22. Lange G, Lu HH, Chang A, Brooks CM. Effect of stretch on the isolated cat sinoatrial node. Am J Physiol 1966;211: 1192-1196.

23. Bolter CP. Intrinsic cardiac rate regulation in the anaesthetized rabbit. Acta Physiol Scand 1994;151:421-428.

24. Barrett CJ, Bolter CP, Wilson SJ. The intrinsic rate response of the isolated right atrium of the rat, Rattus norvegicus. Comp Biochem Physiol A Mol Integr Physiol 1998; 120:391-397.

25. Wilson SJ, Bolter CP. Do cardiac neurons play a role in the intrinsic control of heart rate in the rat? Exp Physiol 2002;87:675-682.

26. Kim D. Novel cation-selective mechanosensitive ion channel in the atrial cell membrane. Circ Res 1993;72:225-231.

27. Van Wagoner DR. Mechanosensitive gating of atrial ATPsensitive potassium channels. Circ Res 1993;72:973-983.

28. Ketch T, Biaggioni I, Robertson R, Robertson D. Four faces of baroreflex failure: hypertensive crisis, volatile hypertension, orthostatic tachycardia, and malignant vagotonia. Circulation 2002;105:2518-2523.

29. Goldstein DS, Eldadah B, Sharabi Y, Axelrod FB. Cardiac sympathetic hypo-innervation in familial dysautonomia. Clin Auton Res 2008;18:115-119.

30. Cannon WB. A law of denervation. Am J Med Sci 1939; 737-750.

31. Smith AA, Dancis J. Exaggerated response to infused norepinephrine in familial dysautonomia. N Engl J Med 1964;270:704-707.

32. McCaughey TJ. Familial dysautonomia as an anaesthetic hazard. Can Anaesth Soc J 1965;12:558-568.

33. Stemper B, Bernardi L, Axelrod FB, Welsch G, Passino C, Hilz MJ. Sympathetic and parasympathetic baroreflex dysfunction in familial dysautonomia. Neurology 2004;63: 1427-1431. 